\title{
Fuzzy knowledge-based approach to treating uncertainty in inventory control
}

\author{
Dobrilla Petrovic* and Edward Sweeney
}

Invenory control in complex manufacturing environments encounters various sources of uncertainity and imprecision. This paper presents one fuzzy knowledge-based approach to solving the problem of order quantity determination, in the presence of uncertain demand, lead time and actual inventory level. Uncertain data are represented by fuzzy numbers, and vaguely defined relations between them are modeled by fuzzy if-then rules. The proposed representation and inference mechanism are verified using a large numbers of examples. The results of three representative cases are summarized. Finally a comparison between the developed fuzzy knowledge-based and traditional, probabilistic approaches is discussed.

Keywords: inventory control, fuzzy set, knowledge-based system, approximate reasoning

A large number of production and inventory models have been developed in the past 40 years, ranging from the simple extensions of classical EOQ (economic order quantity) to complex multi-level inventory models, and from deterministic to stochastic models that represent and operate with uncertain data. The significant differences between models' performances for deterministic and uncertain environments are indicated in the literature. In inventory management, uncertainty arises both in the market place and during the production cycle. In the real world they often occur simultaneously. Demand uncertainty arises due to the possible changes in the order date or in the quantity specified. Supply uncertainty can be caused by a number of factors. For example, supplier lead times could be different from those planned for, or the quantity delivered by vendors could be less than the quantity ordered due to limited availability.

Conventional approaches for treating uncertainty in inventory control are focused on probability theory. It offers a strict, well developed and mathematically attractive apparatus. Despite the large number of models developed that involve probability concepts, there still exists a gap between theory and practice. Some of the problems in their applicability in real inventory problems can highlighted as follows:

1. Uncertain data that describe inventory stock are represented by random variables. Very often, to maintain analytic tractability, three classes of assumptions are made: (a) particular form of the distribution (usually, uncertain demand is modeled by normal, lognormal, Gamma or Poisson distribution); (b) parameters of the distribution are known; and (c) stationarity is assumed, even when parameters are really changing with time or special distributions are used for intermittent or erratic data.

2. To combine various uncertain data, complex distributions are included. For example, daily demand is often modeled by a compound distribution consisting of binomial probabilities of demand occurring on any day and normally distributed quantities when demand occurs. Also, if random varibales are uncorrelated, a bivariable distribution is defined. For example, the resultant lead time demand per unit time distribution is made up of a demand per unit time distribution is made of a demand per unit time distribution interacting within lead time distribution. Those combinations of uncertain data inevitably lead to complex formula and calculations.

3. Modern inventory sock includes a large number of item types. As a result, the amount of required data and computing time for complex procedures are both increased. The problem is usually solved by introducing some assumptions. At the same time, it influences the applicability of the model and reduces the cases for which the model could be successfully used. 
4. Many different lot-sizing techniques are available for order sizing. The choice of lot-sizing rule has a significant effect on the model's performance in the presence of uncertainty.

5. Complex, analytical approaches could be successfully replaced by simulation. Analysis of simulation results, especially for non-terminating inventory control system, requires a large number of simulation runs. Since the starting conditions can create a bias in the estimate of the system's steadystate performance, it is necessary to make the run as long as possible.

Various new methods for treating uncertainty have been developed. They consider uncertainty and imprecision from difficult points of view. The question of the appropriateness of these methods for representing and handling uncertainties in a real, complex inventory control arises. It as attracted attention, both from the viewpoint of theoretical basis and of practical application.

This paper presents a new approach to treating uncertainty in inventory control based on fuzzy set theory. The inventory under consideration is a single-point stock, periodically reviewed. The problem is to determine the replenishment quantities that should be ordered for various types of inventory items.

\section{Fuzzy knowledge-based system}

The quantity that should be ordered far an inventory item depends upon various parameters such as demand, lead time, replenishment rate, required service level and various costs. Most of those parameters could be characterized as follows:

1. Parameter values and relations between them are uncertain and imprecise. Their estimation is often based on the subjective beliefs of managers.

2. It is difficult to measure them, either because there is no unit of measurement or there is no quantitative criterion for representing their values.

3. The knowledge available about their values and relations is incomplete.

4. Some of them are vaguely and unclearly defined.

In this approach, an inventory item is defined by three uncertain parameters: demand, lead time and actual inventory stock level. In practice, they are often imprecisely estimated. For example, expected demand may be expressed as about 10 items, inventory level could be approximately 12 items, lead time about 50 hours or more, and so on. Those approximate qualifiers are presented by fuzzy if-then rules. Fuzzy rules relate angular membership functions are often used in applications, because it models linguistic terms about, more or less, approximately quite well and in a natural way, and it also simplifies the operations on fuzzy numbers. Triangular fuzzy number are determined by three values [l, $\mathrm{m}, \mathrm{u}]$, where $l$ represents the lower bound, $m$ the mean value and $u$ the upper bound of a fuzzy number. The membership degrees are 0 for points $l$ and $u$, and it reaches 1 for $m$. The larger the difference $u-l$, the greater the uncertainty in the represented data. Boundaries are subjective in nature, and are input by the user of the system. Examples of membership functions for uncertain demand, actual inventory level and lead time are given in Figure 1.

Heuristics used to estimate necessary order quantity are presented by fuzzy if-then rules. Fuzzy rules relate dominance between the linguistically described demand and actual inventory level, an imprecise lead time, to the recommended order quantity. The term 'dominance' denotes the result of comparison of demand and inventory level. It denotes the degree to which the demand is higher than the inventory level, such as low, medium and high, and to describe lead time such as short, medium and long. Order quantity could be specified as very small, small, medium, large and very large. All qualifiers are represented by fuzzy sets. Their membership functions are derived by the inventory control exports. The appropriate domain range for lead time and order quantity qualifiers are dependent upon the particular inventory stock. Subjectively defined fuzzy sets for those parameters are shown in Figure 2.

If-then fuzzy rules are defined for every combination of qualifiers. For example, three rules for low dominance between demand and inventory level suggested are:

IF dominance is low AND lead time is short THEN order very small item quantity IF dominance is low AND lead time is medium THEN order small item quantity 
IF dominance is low AND lead time is long THEN order medium item quantity

The rules should reflect the actual inventory policy, and they ban be modified easily.

\section{Approximate reasoning}

Approximate reasoning is the process by which an imprecise conclusion is deduced from a collection of imprecise premised. In this approach, order quantity is estimated according to the imprecise premises of the rules and uncertain data that define the inventory item (i.e. demand, lead time and actual item inventory level). Reasoning is performed through the following steps:

Step 1 Dominance between fuzzy numbers $D$ and $I$ (actual inventory level) is calculated. Various procedures for fuzzy number comparison have been developed. One efficient procedure is applied in this approach. Dominance is a number from the $[0,1]$ interval, where 0 indicates complete nondominance and 1 total dominance of $D$ and $I$. The following formula is used:

Dominance $(\mathrm{D}, \mathrm{I})=$

area where $D$ daminates $I+$ area where $D$ and $I$ are indifferent area of $D+$ area of $I$

The area where D dominates I is defined for two cases:

1. If $\mathrm{D}$ and I do not overlap, the area of dominance is the whole area of $\mathrm{D}$ if the fuzzy number $\mathrm{D}$ is to the right-hand side of the fuzzy number (see Figure 3a).

2. In the case of overlap between $\mathrm{D}$ and I, the area of dominance includes the area that belongs to $\mathrm{D}$ and is to the right-hand side of the overlap and the area that belongs to I and is to the left-hand side of the overlap (see Figure 3b).

The area where $\mathrm{D}$ and I are indifferent is the intersection of the fuzzy numbers $\mathrm{D}$ and I.

Dominance between D and I is 0 or 1 , whenever D and I do not overlap and D is to the left-hand or

right-hand side of I, respectively. Those two extreme cases are covered by the separate heuristic rules.

Step 2 The first fuzzy rule from the knowledge-base is extracted. The rule has the following form:

IF dominance is FD AND lead time is FLT THEN order quantity is FOQ

Where FD, FLT and FOQ are fuzzy qualifiers for dominance, lead time and order quantity size, respectively.

Step 3 The membership degree of dominance (D,I) (calculated in Step 1) in the fuzzy set FD is determined.

Step 4 The item lead time represented by fuzzy number LT and fuzzy qualifier FLT are compared. Compatibility between them is represented by a number in the $[0,1]$ interval, where 0 means total incompatibility between the two fuzzy sets and 1 denotes full compatibility. The following formula is used when the qualifier FLT is medium:

Compatibility $(\mathrm{LT}, \mathrm{FLT})=$
$1-\frac{H(L T, F L T)}{\text { area of } L T+\text { area of } F L T}$

where H(LT, FLT) is the Hamming distance, which measures the difference between two fuzzy sets:

$H(L T, F L T)=f(x a S)[u L T(x)-u F L T(x)] d x$

where $S$ is the domain of fuzzy sets and uLT and uFLT are membership functions for fuzzy lead time and the fuzzy qualifier medium, respectively (see Figure 4b):

compatibility $(\mathrm{LT}, \mathrm{FLT})=$ 
$1-\frac{\text { area of } 2 T \text { intersection of } F L T \text { and } L T}{\text { area of } L T}$

Step 5 To evaluate the rule-premise truth value, the membership value obtained in Step 3 and the compatibility value from Step 4 are combined by the AND connective. The usual definition for AND connective is applied, i.e. a AND $b=\min (a, b)$, where $a$ and $b$ are truth values.

Step 6 The truth value of the rule-consequent part cannot exceed the premise truth value. The appropriate fuzzy set FOQ is 'cut' by the premise truth value. Steps (2)-(6) are repeated for every fuzzy rule in the knowledge-base.

Step 7 The union of the all 'cut' consequent fuzzy sets is found. The union represents the fuzzy quantity order suggested.

Step 8 Translation of the fuzzy described quantity to executable decision is performed by applying arithmetic defuzzification. The moment method is used, i.e. projection of the centre of the area under the membership function curve to the $\mathrm{x}$-axis is selected as a fuzzy set representative scalar. This implies that small premise truth value variations cause continuous changes of recommended order quantity.

\section{Performance analysis}

The approach developed was verified using numerous examples. It was shown that the fuzzy knowledge based system generated outputs which were compatible with the experience of experts in the inventory domain, and were in accordance with common sense knowledge. The influence of various linguistically described inputs to the recommended order quantity was examined.

Three representative test examples are presented. The data common for all test examples were the following: current inventory level I was about 12 items and was modeled by fuzzy number $[10,12,14]$ (see Figure $1 \mathrm{~b}$ ) and qualifiers for dominance between demand and actual inventory level, lead time and order size were defined in Figure 2.

Test 1

Test 1 examined the impact of increasing demand on the recommended order quantity. Lead time was represented by the fuzzy number $[40,50,60]$ hours. Uncertain demand was increasing from about 9 to about 15 items. The results obtained by developed fuzzy knowledge-based system are presented in Table 1.

Increasingly demand causes non-linear order quantity increments. This is compatible with traditional approaches. Abrupt changes in order quantity were notices for demands with mean values in $[10,11]$ and $[13,14]$ intervals. Abruptness is caused by increasing demand dominance over actual inventory level, which is shifted from low to medium to high dominance, respectively.

Table 1: The impact of increasing demand of order quantity

\begin{tabular}{|l|r|r|}
\hline Demand D & Dominance $(\mathbf{D}, \mathbf{l})$ & Order quantity \\
\hline$[5,9,13]$ & 0.125 & 2.333 \\
\hline$[6,10,14]$ & 0.222 & 2.397 \\
\hline$[6.7,10.7$, & & \\
\hline 11.7$]$ & 0.307 & 4.955 \\
\hline$[7,11,15]$ & 0.347 & 9.528 \\
\hline$[8,12,16]$ & 0.5 & 11 \\
\hline$[9,13,17]$ & 0.652 & 11.209 \\
\hline$[9.2,13.2$, & & \\
\hline 17.2$]$ & 0.68 & 15.825 \\
\hline$[10,14,17]$ & 0.778 & 20.804 \\
\hline$[11,15,19]$ & 0.875 & 20.889 \\
\hline
\end{tabular}


Test 2

Test 2 analyzed

sensitivity of the developed approach to the variation of input data uncertainty. Mean value of fuzzy demand was fixed to 14 items. Demand uncertainty was changed by lower and upper bound alterations. Approximate lead time was $[40,50,60]$ hours. The results of the test are given in Table 2.

The output order quantity does not always respond to small changes in the uncertain input demand. The order quantity remains the same as long as the input lead time influences the rule-premise truth value more than demand data. In this test, the following rule was fired:

IF dominance is high AND lead time is medium THEN order high item quantity

The compatibility between the lead time about 50 hours (i.e. fuzzy number $[40,50,60]$ ) and medium lead time was 0.5 . as long as the dominance of demand over actual inventory level was high with the truth value greater than 0.5 , the premise truth value remained 0.5. The minimum function that defines the AND logical connective makes the model more robust. The output can be more sensitive to small input charges by taking over AND connective makes the model more robust. The output can be made more sensitive to small input changes by taking other AND connective definitions (for example $a$ AND $\mathrm{b}=\mathrm{a} \times b$, where $a$ and $b$ are truth values).

\section{Test 3}

In test 3 , both input data were changes. Uncertain demand was increasing from about 10 to about 14 items, and lead time was decreasing from about 90 to about 10 hours. Final order quantities are presented in Table 3.

In accordance with common sense expectations, the calculated order quantities are approximately fixed at the same level- 11 items.

\section{Fuzzy versus probabilistic approach}

The fuzzy knowledge-based approach exhibits some advantages in solving inventory control problems over the traditional probabilistic approach. They can be summarized as follows:

1. The Fuzzy approach offers a more natural description of uncertain data than probability theory.

Linguistic qualifications of unknown data such as demand and lead time are frequently encountered in real-life inventory settings.

Table 2: Sensitivity of order quantity to various levels of demand uncertainty

\begin{tabular}{|l|r|r|}
\hline Demand D & Dominance (D,I) & Order quantity \\
\hline$[13,14,15]$ & 0.94 & 20.889 \\
\hline$[13,14,16]$ & 0.952 & 20.889 \\
\hline$[12,14,15]$ & 0.775 & 20.793 \\
\hline$[12,14,16]$ & 0.875 & 20.889 \\
\hline$[11,14,17]$ & 0.82 & 20.889 \\
\hline$[10,14,18]$ & 0.778 & 20.804 \\
\hline$[9,14,19]$ & 0.745 & 20.671 \\
\hline$[8,14,20]$ & 0.719 & 20.559 \\
\hline$[7,14,21]$ & 0.698 & 19.635 \\
\hline
\end{tabular}


Table 3: Impact of increasing demand and decreasing lead time on order quantity

\begin{tabular}{|r|l|r|l|}
\hline Dominance $(\mathbf{D}, \mathbf{I})$ & Lead time & Order quantity & Demand D \\
\hline 0.778 & $\begin{array}{l}{[80,90,} \\
100]\end{array}$ & 11 & {$[6,10,14]$} \\
\hline 0.719 & {$[70,80,90]$} & 10.384 & {$[6.5,10.5,14.5]$} \\
\hline 0.653 & {$[60,70,80]$} & 11.629 & {$[7,11,15]$} \\
\hline 0.58 & {$[50,60,70]$} & 11 & {$[7.5,11.5,15.5]$} \\
\hline 0.5 & {$[40,50,60]$} & 11 & {$[8,12,16]$} \\
\hline 0.42 & {$[30,40,50]$} & 11 & {$[8.5,12.5,16.5]$} \\
\hline 0.347 & {$[20,30,40]$} & 11.78 & {$[9,13,17]$} \\
\hline 0.281 & {$[10,20,30]$} & 11.979 & {$[9.5,13.5,17.5]$} \\
\hline 0.222 & {$[0,10,20]$} & 11 & {$[10,14,18]$} \\
\hline
\end{tabular}

2. The inventory control strategy can be adequately captured by the fuzzy if-then rules. Fuzzy rules are very flexible, and can be easily modified according to the current control policy.

3. Other parameters that describe complex inventory environments such as replenishment rate, various restrictions concerning warehouse space, production capacity, and so on, could be simply incorporated into fuzzy knowledge-base. On the other hand, in traditional approach, every parameter which is added requires either a new probabilistic model or makes the existing one more complex.

4. Some of the parameters are vaguely defined. For example, linguistically estimated requirements for service level (very high, high, average, and so on) are more appropriate and conceivable than a precisely expressed probability of not running out of stock.

5. It is easier to provide a useful explanation to an end-user of the developed system, and to describe the heuristics and approximate reasoning applied for decision making.

However, possible weaknesses concerning the application of fuzzy sets in general, and in inventory control problems specifically, still remain:

1. Various uncertain data can be represented by triangular membership functions, but there is no strict procedure for creating the appropriate triangular boundaries.

2. There is no guidance for a 'best' selection of logical connective definitions for a given domain specific problem. 


\section{Linguistic qualifiers for lead time: short,}

medium, large

\section{-}

Linguistic qualifiers for order quantity: very small, small medium, large, very large

D-fuzzy demand

I-Fuzzy inventory level LT-fuzzy lead time

Calculate dominance (D,I)

NO

If rule in the knowledge base exists

YES

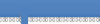

Extract rule IF dominance is FD AND lead time is FLT Them order quantity is FOQ

\section{Determine truth d- membership degree of} dominance $(D, I)$ in the fuzzy set FD

\section{Calculate compatibility (LT, FLT)}

Rule- premise truth value $=\min ($ truth_d, compatibility $($ LT,FLT $))$

Calculate the truth value of the rule-consequent i.e "cut" the fuzzy set FOQ

Find the union of all "cut" consequent fuzzy sets

Apply arithmetic defuzzifiation

Figure 5 Proposed procedure chart 


\section{Concluding remarks}

The fuzzy knowledge-based approach proposed is relatively simple and efficient both analytically and computationally. The two main concepts used, linguistic variables and fuzzy if-then rules, can successfully model uncertain and imprecise inventory stock data and vaguely defined relations between them. The results obtained are encouraging in the following ways:

1. The Fuzzy knowledge-based system can be designed for more complex inventory environments that include various uncertain parameters and different multi-stage inventory hierarchies.

2. The fuzzy approach provides a framework that can be applied to various production control problems that include uncertain, imprecise and incomplete knowledge.

The two approaches compared, based on fuzzy set and probability theory, have different concepts and reasoning principles. It seems more appropriate to use them as a complementary, rather than competitive methods. It raises the question of their possible combination, whoch could lead to better and more realistic inventory modeling.

\section{References}

1. Sand, G 'Predicting demand on the secondary echelon: A case study', in L B Schwartz (ed.), Multilevel Production/Inventory Control Systems: Theory and Practice, North-Holland, Amsterdam (1981) pp 209-225.

2. Brennan, $\mathbf{L}$ and Gupta, $\mathbf{S} \mathbf{M}$ 'A structures analysis of material requirements planning systems under combined demand and supply uncertainty', Int. J. Prod. Res., Vol 31 No 7 (1993) pp 1689-1707.

3. Silver, E A 'Operations research in inventory management: A review and critique', Operat. Res., Vol 29 No 4 (1981) pp 628-644.

4. Lewis, C D Demand Analysis and Inventory Control, Saxon House and Lexington Books (1975).

5. Bahl, H C, Ritzman, L P and Gupta, J N D 'Determining lot sizes and resource requirements: a review', Operat. Res., Vol 35 No 53(1987) pp 329-345.

6. Henkind, $\mathbf{S} \mathrm{J}$ and Harrison, M C 'An analysis of four uncertainty calculi', IEEE Trans. Sysyt., Man \& Cybern., Vol 18 No5 (1988) pp 700-714.

7. Kacprzyk, J and Staniewski, $\mathbf{P}$ 'Long-term inventory policy-making through fuzzy cesicion-making models', Fuzzy Sets and Syytems, Vol 8 (1982) 117-132.

8. Zadeh, L A 'A theory of approximate reasoning', in J E Hayes, D Michie and L J Mikulich (eds.), Machine Intelligence 9, Wiley, New York (1979) pp 149-194.

9. Tseng, $\mathbf{Y}$ T and Klein, $\mathbf{C} \mathbf{M}$ 'New algorithm for the ranking procedure in fuzzy decision making', IEEE Trans. Syst., Man \& Cybern., Vol 19 No 5 (1989) pp 1289-1296

10. Dubois, D and Prade, H Fuzzy Sets and Systems: Theory and Applications, Academic Press, New-York (1980).

11. Grahm, I and Jones, P L Expert Systems, Knowledge, Uncertainty and Decision, Chapman and Hall, London (1988). 